https://doi.org/10.15407/ujpe65.2.106

D. FISCALETTI, ${ }^{1}$ A. SORLI ${ }^{2}$

${ }^{1}$ SpaceLife Institute

(Italy; e-mail: spacelife.institute@gmail.com)

${ }^{2}$ Foundations of Physics Institute

(Slovenia; e-mail: sorli.amrit@gmail.com)

\title{
A THREE-DIMENSIONAL NON-LOCAL QUANTUM VACUUM AS THE ORIGIN OF PHOTONS
}

\begin{abstract}
A model of a three-dimensional quantum vacuum defined by the processes of creation/annihilation of quanta corresponding to elementary energy density fluctuations is proposed. In it, a photon is not a primary physical reality but emerges itself as a special state of the threedimensional quantum vacuum. In this model, the three-dimensional quantum vacuum has a ground state which acts as a "cosmic reservoir" of photons, which emits and absorbs photons and Planck's law of the spectral distribution of the energy radiated by a black body derives from the fundamental processes in the three-dimensional quantum vacuum, in particular, in the context of a quantization volume responsible for the appearance of photons. Finally, the idea of the Lamb shift of hydrogenoid atoms as a phenomenon determined by the ground state of the quantum vacuum which acts as a reservoir of photons is explored.

Ke ywords: black body radiation, CMB, photon, quantum vacuum, fluctuations of the quantum vacuum energy density, reduction-state processes of creation/annihilation of quanta.
\end{abstract}

\section{Introduction}

CMB (cosmic microwave background) is supposed to be the relict radiation which started after the Big Bang in the recombination period. The CMB is characterized by an anisotropy which is of crucial physical importance in the sense that, in correspondence to tiny temperature fluctuations, small density inhomogeneities occur in $\mathrm{CMB}$, grow in the time under the influence of the gravity, and become all the structures we see now in the Universe. Without an anisotropic $\mathrm{CMB}$, we would not exist. But what was the origin of these small fluctuations?

The simple calculation shows that CMB could not reach us yet. The diameter of the Universe is 13.7 billion light years according to the Big Bang model. The Universe radius measured on the basis of astronomic observations is 46.6 billion light years. This means

(C) D. FISCALETTI, A. SORLI, 2020 that CMB has reached the distance from the hypothetical Big Bang which is about 13.7 billion light years and is now 32.9 billion light years far from us [1]. By taking the fact above, CMB has origin in a quantum vacuum characterized by energy fluctuations. We suggest the possibility that the real origin of the small temperature fluctuations associated with the small density inhomogeneities characterizing CMB is represented just by the elementary energy fluctuations of the quantum vacuum.

The quantum vacuum can be considered as the "cosmic reservoir" of all the spectrum of electromagnetic radiation. When the quantum vacuum is heated up, the spectrum of radiation is extending. Our Sun is emitting the entire electromagnetic spectrum [2].

Every physical object is emitting the "black body radiation". The ultimate origin of this radiation is the quantum vacuum. When a given body is absorbing a given photon radiation, photons are not stored in elec-

ISSN 2071-0194. Ukr. J. Phys. 2020. Vol. 65, No. 2 
trons; they are stored back into the quantum vacuum as a background which pullulates of the vibrating energy and vitality.

Let us consider, for example, two plates of iron. Let us heat the left plate, so that it starts glowing and emitting photons (see Fig. 1). When we heat the left plate, this perturbs the activity of the quantum vacuum in the sense that photons in the quantum vacuum are getting excited. As a consequence, the right plate absorbs these photons which are emitted from the left plate. By virtue of these processes, the electrons of the material tend to jump on the higher energy levels. Each electron is excited by a specific photon, and, after hitting its corresponding electron, each given photon is dissolving back into the vacuum.

In the famous pioneering work of 1945, Wheeler and Feynman proposed the idea of that the oscillation of an electron provokes the reaction of all the other charged particles through the transmission of direct signals by each particle at a distance without the action of fields [3]. In this approach known as the Wheeler-Feynman absorber theory, the medium represented by the total effect of all the things existing in the Universe is a perfect absorber as regards the received signal and, at the same time, is a perfect emitter as regards the emitted signal. Then, in a series of papers published during the 1980s [4-7], by following the philosophy of the Wheeler-Feynman absorber theory, J. Cramer developed an interpretation of quantum mechanics, the so-called transactional theory. In it, the view of the action at distance between electrons implies the possibility to define a vacuum background characterized by a fundamental non-locality, where a quantum process is a timesymmetric process, in which one has an "absorber" which generates confirmation waves in response to an emitted offer wave. In recent years, the transactional theory of J. Cramer was finally extended into a real fledged theory, on one hand, by Ruth Kastner [8-11]. He built a possible transactional interpretation, where the space-time unfolds as an emergent manifold from a more fundamental collective structure, a "pre-space-time" characterized by "transactional processes" (constituted by the emission and absorption of quanta) involving de Broglie waves and resulting in transfers of energy from an emitter to an observer, which must be considered as pre-spacetime objects at the micro-level. On the other hand, I. Licata and L. Chiatti [12-17] developed an ap-

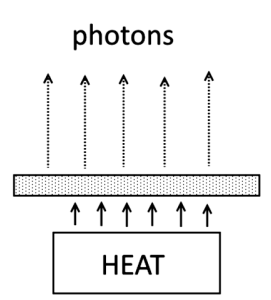

photons are emitted from vacuum

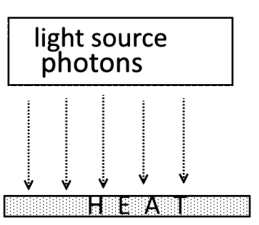

photons are absorbed into vacuum
Fig. 1. Photon emission and absorption

proach, where the fundamental arena of the Universe is an archaic, timeless vacuum from which patterns emerge by reduction-state processes corresponding to the events of creation and destruction (or, in other words, the physical manifestation and demanifestation) of certain quantities, and such patterns influence the vacuum activity, in a quantum feedback.

Now, according to the model of a three-dimensional (3D) timeless quantum vacuum proposed by the authors of this paper in [18-25], it is possible to go beyond the results of Wheeler, Feynman, Cramer, Kastner, Chiatti, and Licata in the following sense. The real key of the explanation of the direct interaction at a distance between material particles and their medium invoked by the Wheeler-Feynman approach, as well as of the transactional processes considered by Cramer, Kastner, Chiatti, and Licata, lies in the fact that there is a fundamental symmetry between matter and space. In our approach, in fact, matter and space are made by the same "stuff": subatomic particles are given by opportune diminutions of the quantum vacuum energy density with respect to the Planck energy density

$\rho_{\mathrm{P} E}=\frac{m_{\mathrm{P}} c^{2}}{l_{\mathrm{P}}^{3}}=4.641266 \times 10^{113} \mathrm{~J} / \mathrm{m}^{3}$

(where $m_{\mathrm{P}}$ is Planck's mass, $c$ is the light speed, and $l_{\mathrm{P}}$ is Planck's length), which represents the ground state of the 3D quantum vacuum, corresponding to elementary reduction-state (RS) processes of creation/annihilation of quanta of the $3 \mathrm{D}$ quantum vacuum which are somewhat similar to the transactional processes invoked by Chiatti and Licata [12-17] in their interpretation of an archaic, atemporal vacuum. In other words, the elementary particles and massive bodies in our model are just different structures of the quantum vacuum. Every elementary particle can be seen as an extended form of the quan- 


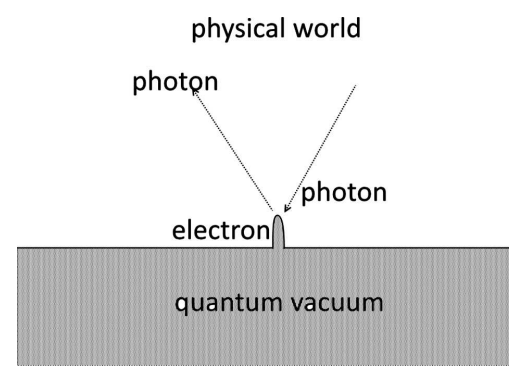

Fig. 2. Electron as the door of exit and entrance of a photon

tum vacuum which appears in the physical world as a "particle". We note that the original WheelerFeynman absorber theory, allowing an interpretation of light as the direct and instantaneous interaction between electrons, eliminated photons as a physical reality. But in our model of 3D timeless quantum vacuum, we can say that electrons are not emitting and absorbing photons on their own. Electrons are quantum vacuum structures through which photons are emitted from the vacuum and absorbed into the vacuum as a consequence of the more fundamental elementary RS processes of creation/annihilation (see Fig. 2 below). This means that photons cannot be considered as primary physical realities, but are emergent from the network of RS processes of creation/annihilation generating the fluctuations of the quantum vacuum energy density. Photons are ultimately generated by the fundamental processes of the $3 \mathrm{D}$ quantum vacuum.

This paper is structured in the following way. In Section 2, we will make some preliminary considerations about a 3D quantum vacuum as the fundamental arena of the Universe in a bijective research methodology. In Section 3, we will develop our new suggestive interpretation of the photon inside the $3 \mathrm{D}$ timeless quantum vacuum. In Section 4, we will analyze how to re-read Planck's law of spectral distribution of the energy radiated by a black body in our model of the 3D quantum vacuum. Finally, in Section 5, we will show in what sense the 3D quantum vacuum acting as a reservoir of photons is the primary physical reality which is responsible for the Lamb shift of hydrogen-like atoms.

\section{Bijective Research Methodology on Quantum Vacuum}

In the 20-th century, the prevalent paradigm in physics was that particles and fields are different types of energy, which exists in an "empty space". Following P. Dirac, the physical vacuum is not seen as a purely geometrical container, and the concept of virtual particles populating the physical vacuum and the idea of vacuum polarization have become a standard way to consider the physical vacuum. Indeed, the Standard Model of particle physics yet does not account for the quantum features of the vacuum, in particular, its energy density, and yet does not manage to understand the real physical meaning of the virtual particles of the vacuum.

In the 21-st century, the understanding has appeared that the existence of energy in the space requires that the space also be a type of energy. According to the bijective research methodology proposed by the authors of this paper, which requires the existence of a direct epistemological correspondence between elements of a theory and elements of the physical reality, the universal space has the origin in a $3 \mathrm{D}$ quantum vacuum which is the primordial energy of the Universe and fundamental arena of the Universe [26]. We can denote the physical quantum vacuum as a set $X_{0}$ and a model of the quantum vacuum as a set $Y_{0}$. In the set $X_{0}$, we have different types of energy $X_{1}, X_{2}, X_{3}, \ldots, X_{n}$. In the set $Y_{0}$, we also have the corresponding different types of energy $Y_{1}, Y_{2}, Y_{3}, \ldots, Y_{n}$. The sets $X_{0}$ and $Y_{0}$ are related by the bijective function:

$$
\begin{aligned}
& f: X_{0} \rightarrow Y_{0}, \\
& f:\left\{X_{1}, X_{2}, X_{3}, \ldots, X_{n}\right\} \rightarrow\left\{Y_{1}, Y_{2}, Y_{3}, \ldots, Y_{n}\right\} .
\end{aligned}
$$

The bijective methodology is assuring a 100-\% exact model of physical reality, because every element in the model corresponds exactly to one element in the physical world. According to the bijective methodology, the primitive ontology of our $3 \mathrm{D}$ quantum vacuum model is thus represented by the different energetic fluctuations of the quantum vacuum occurring in different regions, their relations of distance, and their changes. All the other constructions of physics are somewhat emergent with respect to the network of these energetic fluctuations, their relations of distance, and their evolution. In particular, the quantum vacuum in this model is the "cosmic reservoir" of photons and appears as a kind of the Einstein-Bose condensate.

In particular, the bijective model implies that the notion of force cannot be considered as a primary 
physical reality in our physical theories, but is an emerging property which derives from the motion of a moving object. In other words, force does not have existence on its own. From the point of view of the bijective methodology, one can say that the different physical fields and interactions can be seen as different aspects of the same 3D timeless quantum vacuum, which is the only field existing in the Universe at a fundamental level.

As regards electromagnetism, the frequency of the virtual radiation generated by the motion of virtual particles-antiparticles associated with the evolution of the RS processes of the $3 \mathrm{D}$ quantum vacuum may be considered as the origin of the electromagnetic effects of the 3D quantum vacuum. In particular, the electromagnetic field inside a perfectly reflecting cavity is associated with the infinite different modes of the fundamental 3D quantum vacuum, where each mode corresponds to an independent oscillation defined by the frequency produced by a specific RS process of creation/annihilation of quanta in correspondence to elementary fluctuations of the $3 \mathrm{D}$ quantum vacuum.

\section{From the Reduction-State Processes of Creation/Annihilation to a Photon as a Wave-Corpuscle Emerging Entity of the Three-Dimensional Quantum Vacuum}

The 3D quantum vacuum is a sort of the Bose-Einstein condensate acting as a superfluid medium in the sense that the RS processes of creation/annihilation of virtual particle/antiparticle pairs give rise to a total zero spin. The excited state of the 3D quantum vacuum corresponding to the appearance of a material particle of mass $m$ is defined (at the center of that particle) by the energy density

$\rho=\rho_{p E}-\frac{m c^{2}}{V}$

where $V$ is the volume of the massive particle. In other words, each subatomic particle is generated by diminishing the quantum vacuum energy density with respect to the Planck energy density (1) characterizing the ground state. The evolution of the opportune RS processes of creation/annihilation of particle/antiparticle pairs generating a particle is described by a wave function $C=\left(\begin{array}{l}\psi \\ \phi\end{array}\right)$ at two com- ponents satisfying a time-symmetric extension of the Klein-Gordon quantum relativistic equation

$\left(\begin{array}{cc}H & 0 \\ 0 & -H\end{array}\right) C=0$,

where $H=\left(-\hbar^{2} \partial^{\mu} \partial_{\mu}+\frac{V^{2}}{c^{2}}\left(\Delta \rho_{q \nu E}\right)^{2}\right)$ and $\Delta \rho_{q \nu E}=$ $=\left(\rho_{\mathrm{P} E}-\rho_{q \nu E}\right)$ is a change of the quantum vacuum energy density [18-25]. Equation (5) corresponds to the following two equations:

$\left(-\hbar^{2} \partial^{\mu} \partial_{\mu}+\frac{V^{2}}{c^{2}}\left(\Delta \rho_{q \nu E}\right)^{2}\right) \psi_{Q, i}(x)=0$

for creation events and

$\left(\hbar^{2} \partial^{\mu} \partial_{\mu}-\frac{V^{2}}{c^{2}}\left(\Delta \rho_{q \nu E}\right)^{2}\right) \phi_{Q, i}(x)=0$

for destruction events. Moreover, the RS processes are choreographed by a quantum potential of the vacuum

$Q_{Q, i}=\frac{\hbar^{2} c^{2}}{V^{2}\left(\Delta \rho_{q \nu E}\right)^{2}}\left(\begin{array}{c}\frac{\left(\nabla^{2}-\frac{1}{c^{2}} \frac{\partial^{2}}{\partial t^{2}}\right)\left|\psi_{Q, i}\right|}{\left|\psi_{Q, i}\right|} \\ -\frac{\left(\nabla^{2}-\frac{1}{c^{2}} \frac{\partial^{2}}{\partial t^{2}}\right)\left|\phi_{Q, i}\right|}{\left|\phi_{Q, i}\right|}\end{array}\right)$

which makes the 3D quantum vacuum as a nonlocal manifold. In the light of the quantum potential (8) of the vacuum, the $3 \mathrm{D}$ quantum vacuum characterized by fluctuations of its energy density can be interpreted as an ultimate immediate medium of quantum entanglement, the fundamental arena, and the fundamental source of non-local phenomena. In this picture, the time is not a fundamental reality in which material changes occur, but exists only as a mathematical parameter which measures the sequential numerical order of motion of local objects which takes place in the fundamental non-local $3 \mathrm{D}$ quantum vacuum [27]. Moreover, in the original Wheeler-Feynman absorber theory and in our 3D quantum vacuum approach, one deals with oscillations of the vacuum corresponding to the RS processes of creation/annihilation of quanta which act instantaneously at a distance one with another. and the fundamental entity which rules this instantaneous action at a distance is the non-local quantum potential of the vacuum at two components (8).

As a consequence of the motion of the virtual particles corresponding to the elementary fluctuations of 
the quantum vacuum energy density, the space-time turns out to be filled with the virtual radiation with frequency

$\omega=\frac{2 \Delta \rho_{q \nu E} V}{\hbar n}$,

where $n$ is the number of the RS processes of virtual subparticles characterizing the vacuum medium. The quantum potential (8) of the vacuum choreographs, in a non-local way, the appearance of oscillations (9) associated with the RS processes. In particular, if one uses the language of the original Wheeler-Feynman absorber theory, one can say that the first component of the quantum potential (8) of the vacuum expresses the fact that the $3 \mathrm{D}$ quantum vacuum is a perfect absorber as regards the received signal. The second component of the quantum potential (8) of the vacuum expresses the fact that the 3D quantum vacuum is a perfect emitter as regards the emitted signal.

In our model, the electromagnetic properties of nature emerge from the primitive ontology of the theory, namely the RS processes of creation/annihilation of quanta corresponding to fluctuations of the quantum vacuum energy density, as a consequence of the action of oscillations (9) generated by these RS processes. Frequency (9) may be considered as the origin of the electromagnetic effects of the $3 \mathrm{D}$ quantum vacuum. For example, the electromagnetic field inside a perfectly reflecting cavity can be seen as an expansion of infinite different modes of the fundamental 3D quantum vacuum, where each mode corresponds to an independent oscillation defined by frequency (9) produced by a specific RS process of creation/annihilation of quanta in correspondence to elementary fluctuations of the $3 \mathrm{D}$ quantum vacuum. This means that the zero-point energy density defining the electromagnetic effects of the $3 \mathrm{D}$ quantum vacuum is determined by fluctuations of the quantum vacuum energy density and by the quantum potential corresponding to the RS processes on the basis of the relation

$W_{\rho_{q \nu E}}=8 \frac{\left(\Delta \rho_{q \nu E}\right)^{3} V^{3} Q_{Q, i}}{\hbar^{3} \pi n^{3} c^{3}}$.

By starting from Eqs. (9) and (10), we demonstrated in [21] that the electric and magnetic properties of the quantum vacuum and, thus, the electromagnetic field of the space can be directly derived, on one hand, by considering the semiclassical approach known as stochastic electrodynamics (SED) and, on the other hand, inside quantum electrodynamics (QED). According to our results, the electric and magnetic fields derived from the zero-point fluctuations of the $3 \mathrm{D}$ quantum vacuum can be written in the SED approximation as

$$
\begin{aligned}
& \mathbf{E}_{\tau}^{z p}(\mathbf{r}, t)=\sum_{\lambda=1}^{2} \int d^{3} k\left(\Delta \rho_{q \nu E} V / n \pi^{2}\right)^{1 / 2} \times \\
& \times \hat{\varepsilon}(\mathbf{k}, \lambda) \cos \left[\mathbf{k r}-\frac{2 \Delta \rho_{q \nu E} V}{\hbar n} t-\theta(\mathbf{k}, \lambda)\right] \\
& \mathbf{B}^{z p}(\mathbf{r}, t)=\sum_{\lambda=1}^{2} \int d^{3} k\left(\Delta \rho_{q \nu E} V / n \pi^{2}\right)^{1 / 2} \times \\
& \times[\hat{k} \times \hat{\varepsilon}(\mathbf{k}, \lambda)] \cos \left[\mathbf{k r}-\frac{2 \Delta \rho_{q \nu E} V}{\hbar n} t-\theta(\mathbf{k}, \lambda)\right]
\end{aligned}
$$

in a stationary frame (where the sum is over two polarization states, where $\hat{\varepsilon}$ is a unit vector, $\mathbf{k}$ is the polarization vector such that $|\mathbf{k}|=\omega / c$ and $\theta(\mathbf{k}, \lambda)$ is a random variable uniformly distributed in the interval $(0,2 \pi)$ and independently for each wave vector $\mathbf{k}$ and polarization index $\lambda$ ); while, in the Rindler frame (characterized by a constant proper acceleration) and defined by the following velocity and Lorentz factor, respectively,

$\frac{v}{c}=\tanh \left(\frac{a \tau}{c}\right)$,
$\gamma_{\tau}=\cosh \left(\frac{a \tau}{c}\right)$,

where $\alpha$ is object's proper acceleration, and $\tau$ its proper time, they become

$\mathbf{E}_{\tau}^{z p}(0, \tau)=\sum_{\lambda=1}^{2} \int d^{3} k\left(\Delta \rho_{q \nu E} V / n \pi^{2}\right)^{1 / 2} \times$
$\times\left\{\hat{x} \hat{\varepsilon}_{x}+\hat{y} \cosh \left(\frac{a \tau}{c}\right)\left[\hat{\varepsilon}_{y}-\tanh \left(\frac{a \tau}{c}\right)(\hat{k} \times \hat{\varepsilon})_{z}\right]+\right.$
$\left.+\hat{z} \cosh \left(\frac{a \tau}{c}\right)\left[\hat{\varepsilon}_{z}+\tanh \left(\frac{a \tau}{c}\right)(\hat{k} \times \hat{\varepsilon})_{y}\right]\right\} \times$
$\times \cos \left[k_{x} \frac{c^{2}}{a} \cosh \left(\frac{a \tau}{c}\right)-\frac{2 \Delta \rho_{q \nu E} c V}{a \hbar n} \sinh \left(\frac{a \tau}{c}\right)-\theta(\mathbf{k}, \lambda)\right]$,

$\mathbf{B}_{\tau}^{z p}(0, \tau)=\sum_{\lambda=1}^{2} \int d^{3} k\left(\Delta \rho_{q \nu E} V / n \pi^{2}\right)^{1 / 2} \times$

ISSN 2071-0194. Ukr. J. Phys. 2020. Vol. 65, No. 2 
$\times\left\{\hat{x}(\hat{k} \times \hat{\varepsilon})_{x}+\hat{y} \cosh \left(\frac{a \tau}{c}\right)\left[(\hat{k} \times \hat{\varepsilon})_{y}-\tanh \left(\frac{a \tau}{c}\right)(\hat{\varepsilon})_{z}\right]+\right.$ $\left.+\hat{z} \cosh \left(\frac{a \tau}{c}\right)\left[\hat{\varepsilon}_{z}+\tanh \left(\frac{a \tau}{c}\right)(\hat{k} \times \hat{\varepsilon})_{y}\right]\right\} \times$

$\times \cos \left[k_{x} \frac{c^{2}}{a} \cosh \left(\frac{a \tau}{c}\right)-\frac{2 \Delta \rho_{q \nu E} c V}{a \hbar n} \sinh \left(\frac{a \tau}{c}\right)-\theta(\mathbf{k}, \lambda)\right]$,

where $\hat{\varepsilon}_{x}$ is the scalar projection of the $\hat{\varepsilon}$ unit vector along the $x$-direction, and similarly for $\hat{\varepsilon}_{y}$ and $\hat{\varepsilon}_{z}$. Equations (11) and (12) for stationary frames (as well as Eqs. (15) and (16) for Rindler frames)) indicate clearly that the electric and magnetic fields are two different kinds of the polarization of the $3 \mathrm{D}$ quantum vacuum produced by the frequencies of the radiation associated with the motion of virtual particles of the $\mathrm{RS}$ processes, namely, by fluctuations of the quantum vacuum energy density.

In our approach, the photon may be considered as an indivisible "wave-corpuscle" entity extended over a wavelength which emerges from the electromagnetic properties of the 3D quantum vacuum propagating along a vector potential wave function. On the basis of fruitful considerations made by Meis [28, 29], we can propose here that, in our manifest world, a photon is generated by frequency (9) of the $3 \mathrm{D}$ quantum vacuum under opportune conditions, namely, when it corresponds to the minimum "quantum" of the electromagnetic properties of the vacuum characterized by a quantized vector potential which depends on the quantum vacuum energy density fluctuations and the number of the RS processes in the volume under consideration. The quantized vector potential of the $3 \mathrm{D}$ quantum vacuum has the form

$$
\begin{aligned}
& \boldsymbol{\alpha}_{\frac{\Delta \rho_{q \nu E}}{n}}=\xi \frac{2 \Delta \rho_{q \nu E}}{\hbar n}\left[\hat{\varepsilon}_{\lambda} e^{i\left(\mathbf{k} \cdot \mathbf{r}-\frac{2 \Delta \rho_{q \nu E}}{\hbar n} t+\varphi\right)}+\right. \\
& \left.+\hat{\varepsilon}_{\lambda}^{*} e^{-i\left(\mathbf{k} \cdot \mathbf{r}-\frac{2 \Delta \rho_{q \nu E}}{\hbar n} t+\varphi\right)}\right]=\frac{2 \Delta \rho_{q \nu E}}{\hbar n} \boldsymbol{\Xi}_{\frac{\Delta \rho_{q \nu E}}{n}}(\mathbf{r}, t),
\end{aligned}
$$

where we have defined the field

$$
\begin{aligned}
& \boldsymbol{\Xi}_{\frac{\Delta \rho_{q \nu E}}{n}}(\mathbf{r}, t)=\left[\xi \hat{\varepsilon}_{\lambda} e^{i\left(\mathbf{k} \cdot \mathbf{r}-\frac{2 \Delta \rho_{q \nu E}}{\hbar n} t+\varphi\right)}+\right. \\
& \left.+\xi \hat{\varepsilon}_{\lambda}^{*} e^{-i\left(\mathbf{k} \cdot \mathbf{r}-\frac{2 \Delta \rho_{q \nu E}}{\hbar n} t+\varphi\right)}\right],
\end{aligned}
$$

where $\hat{\varepsilon}_{\lambda}$ is a complex unit vector of polarization (which expresses the polarization features of a region of the $3 \mathrm{D}$ quantum vacuum), $\varphi$ is a phase parameter, and $\xi$ is an opportune constant having physical dimensions Volt $m^{-1} s^{-2}$ (we underline that, inside Meis' approach, accounting for Maxwell's equation, the experimental evidence, the experimental results, as well as the normalization of the energy over a wavelength of a classical electromagnetic plane wave with circular polarization to Planck's expression of the quantized radiation energy, this constant turns out to have the value $|\xi| \infty\left|\frac{\hbar}{4 \pi e c}\right| \approx 1.747 \times$ $\times 10^{-25} \mathrm{~V} \mathrm{~m}^{-1} \mathrm{~s}^{-2}$ ). According to Eq. (17), the propagation of a photon corresponding to the quantized vector potential occurs within a period $T$ over a wavelength $\lambda$ and is repeated successively along the propagation axis. In view of Meis' calculations, the quantized vector potential $\frac{\alpha \Delta \rho_{q \nu E}}{n}$ obeys the wave equation

$\nabla^{2} \boldsymbol{\alpha}_{\frac{\Delta \rho_{q \nu E}}{n}}(\mathbf{r}, t)-\frac{1}{c^{2}} \frac{\partial^{2}}{\partial t^{2}} \boldsymbol{\alpha}_{\frac{\Delta \rho_{q \nu E}}{n}}(\mathbf{r}, t)=0$.

Moreover, it satisfies the photon vector potential linear time differential equation

$i \xi \frac{\partial}{\partial t} \boldsymbol{\alpha}_{\frac{\Delta \rho_{q \nu E}}{n}}(\mathbf{r}, t)=\tilde{\alpha}_{0} \boldsymbol{\alpha}_{\frac{\Delta \rho_{q \nu E}}{n}}(\mathbf{r}, t)$,

where $\tilde{\alpha}_{0}=-i \xi c \boldsymbol{\nabla}$ is the vector potential amplitude operator with eigenvalue $\frac{2 \Delta \rho_{q \nu E}}{\hbar n} \xi$, as well as Schrödinger's equation for the energy with the massless Hamiltonian $\tilde{H}=-i \hbar c \boldsymbol{\nabla}$ with eigenvalue $\frac{2 \Delta \rho_{q \nu E}}{n}$ of the form

$i \hbar \frac{\partial}{\partial t} \boldsymbol{\alpha}_{\frac{\Delta \rho_{q \nu E}}{n}}(\mathbf{r}, t)=\tilde{H} \tilde{\alpha}_{\frac{\Delta \rho_{q \nu E}}{n}}(\mathbf{r}, t)$.

By combining Eqs. (20) and (21), one obtains a wave-particle equation for the vector potential and the energy of a photon in the $3 \mathrm{D}$ quantum vacuum:

$i\left(\begin{array}{l}\xi \\ \hbar\end{array}\right) \frac{\partial}{\partial t} \boldsymbol{\alpha}_{\frac{\Delta \rho_{q \nu E}}{n}}(\mathbf{r}, t)=\left(\begin{array}{c}\tilde{\alpha}_{0} \\ \tilde{H}\end{array}\right) \boldsymbol{\alpha}_{\frac{\Delta \rho_{q \nu E}}{n}}(\mathbf{r}, t)$.

Equation (22) indicates that the vector potential $\boldsymbol{\alpha}_{\frac{\Delta \rho_{q \nu E}}{n}}(\mathbf{r}, t)$, which depends on the frequency of virtual particles of the $3 \mathrm{D}$ quantum vacuum and which has a quantized amplitude, plays the role of a real wave function for the photon in a non-local representation. In other words, in the light of Eq. (22), one can say that the quantized vector potential is the fundamental entity which expresses the non-local features of the photons generated in the $3 \mathrm{D}$ quantum vacuum. 
Thus, the probability for detecting a given photon corresponding to a certain fluctuation of the quantum vacuum energy density around the point $\mathbf{r}$ is proportional to the square of the angular frequency

$P_{\frac{\Delta \rho_{q \nu E}}{n}}(\mathbf{r}) \propto \frac{4 \xi^{2} \Delta \rho_{q v E^{2}}}{\hbar^{2} n^{2}}$.

Now, in this picture, the volume of the $3 \mathrm{D}$ quantum vacuum which gives rise to the appearance of a photon is quantized in order to obtain a normalization of the energy over a wavelength of a classical electromagnetic plane wave with circular polarization which is compatible with Planck's expression of the quantized radiation energy. In particular, this requirement leads to the following expression regarding the quantized volume of the $3 \mathrm{D}$ quantum vacuum originating the appearance of a photon:

$V_{\frac{\Delta \rho_{q \nu E}}{n}}=\left(\frac{\hbar}{2 \varepsilon_{0} \xi^{2}}\right)\left(\frac{2 \Delta \rho_{q \nu E}}{\hbar n}\right)^{-3}$.

Equation (24) regarding the quantization of the volume of the 3D quantum vacuum which originates the presence of a photon may be therefore considered as the real physical origin of Planck's blackbody spectrum inside the 3D quantum vacuum model. The energy and momentum of a photon can be defined inside

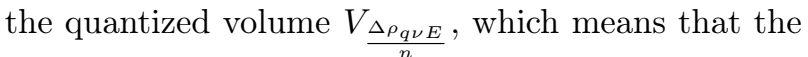
photon here is an emerging entity from more fundamental properties of the $3 \mathrm{D}$ quantum vacuum, which has a minimum spatial extension corresponding to the quantization volume in which the quantized vector potential of the $3 \mathrm{D}$ quantum vacuum oscillates over a period. A photon with wavelength $\lambda_{\underline{\Delta \rho_{q \nu E}}}$ can be detected within a volume of the order of the cube of its wavelength $\lambda_{\frac{\Delta \rho_{q \nu E}}{n}}$.

In summary, the following re-reading of the origin of photons in the 3D quantum vacuum emerges. Elementary RS processes of creation/annihilation of virtual particle/antiparticle pairs corresponding to elementary fluctuations of the quantum vacuum energy density generate oscillations (9) of the vacuum which are choregraphed in a non-local way by the quantum potential of the vacuum. Under opportune conditions, when the region of space is characterized by a certain polarization expressed by the complex unit vector $\hat{\varepsilon}_{\lambda}$, they can give rise to the minimum quantum of the electromagnetic field, which can be characterized in terms of the quantized vector potential (17) satisfying the wave equation (19), the linear time differential equation (20), and the Schrödinger equation (21), which can be thus combined into a wave-particle general equation (22). It implies that the photon is a "wave-corpuscle" entity emerging from more fundamental properties of the non-local 3D quantum vacuum which is a perfect absorber and emitter. If, according to quantum mechanics, a photon or a general quantum system indeed occupies all space before being localized, this fact in our theory can be explained in the light of the non-local features of the 3D quantum vacuum.

Namely, it is the non-local quantum potential of the vacuum that is practically producing a nonlocalization of the particles, as well as implying that oscillations of the vacuum corresponding to the RS processes of creation/annihilation of quanta act instantaneously at a distance one with another, in the spirit of the original Wheeler-Feynman absorber theory and the transactional approach. Moreover, a crucial result of our theory is that the appearance of a photon ultimately derives from a quantized volume given by (24) which can be considered as the real key of re-reading and explanation of the timeless quantum jumps invoked by the Chiatti-Licata theory as regards the interpretation of subatomic particles, as a result of the microscopic processes of nucleation of the vacuum, as the processes of entrance and exit associated to a real microinteraction from the usual temporal domain to a timeless vacuum.

Now, on the basis of the mathematical treatment of photons in the $3 \mathrm{D}$ quantum vacuum represented by Eqs. (17)-(23), a fundamental result is the following. Although when frequency (9) is null, all the physical properties of a photon vanish, it must be observed that, even if $\omega=0$, the field $\boldsymbol{\Xi}_{\frac{\Delta \rho_{q \nu E}}{n}}$ appearing in (17) is not null and becomes the vacuum field $\boldsymbol{\Xi}_{0}$ involving the polarization and generating the electronic transitions in matter. This is a fundamental point: this physically means that, when a region of the $3 \mathrm{D}$ quantum vacuum is described by the quantized vector potential (17) satisfying the fundamental wave-particle equation (22), this region of the vacuum becomes a source of physical (electromagnetic) processes, even if frequency (9) of this region becomes null. It is the vacuum field which is present in this situation that produces the polarization and is responsible for electronic transitions in matter. This vacuum field can be expressed both as a vector field and a quantum

ISSN 2071-0194. Ukr. J. Phys. 2020. Vol. 65, No. 2 
mechanical operator as follows:

$\boldsymbol{\Xi}_{0}=\xi \hat{\varepsilon}_{\lambda} e^{i \varphi}+\xi^{*} \hat{\varepsilon}_{\lambda}^{*} e^{-i \varphi}$,

$\tilde{\Xi}_{0}=\xi a_{k, \lambda} \hat{\varepsilon}_{\lambda} e^{i \varphi}+\xi^{*} a_{k, \lambda}^{+} \hat{\varepsilon}_{\lambda}^{*} e^{-i \varphi}$,

where $a_{k, \lambda}$ and $a_{k, \lambda}^{+}$are the usual annihilation and creation operators of the $k$ th mode, and $\lambda$ is the polarization of a three-dimensional photon, respectively. Equations (25) and (26) define a sort of the "ground state" for the frequency of the 3D quantum vacuum (9) generating photons. The field $\boldsymbol{\Xi}_{0}$ can be considered, also by virtue of the fact that its physical units are $\mathrm{V} \mathrm{m}^{-1} \mathrm{~s}^{2}$, as a real component of the $3 \mathrm{D}$ quantum vacuum, namely, as the special basic state of the 3D quantum vacuum expressing its electromagnetic properties. In other words, the "ground state" of the electromagnetic quantum vacuum is suggested in the model. It is this ground state given by the field $\boldsymbol{\Xi}_{0}$ that is the ultimate source which produces the appearance of photons in the explicate manifesting level of our physical world. This ground state of the frequency of the 3D quantum vacuum (9) generating photons can be considered as a peculiar state of the $3 \mathrm{D}$ quantum vacuum which physically acts as the fundamental "cosmic reservoir" of photons in the sense that it is the primary generating source of photons, even when the energy and vector potential are null. In other words, we can say that the ground state describing the electromagnetic properties of the 3D quantum vacuum, which is defined by Eqs. (25) and (26) represents the ultimate source from which photons are born. This means that, in this approach, while subatomic massive particles are ultimately generated by more elementary physical processes regarding the RS processes of the $3 \mathrm{D}$ quantum vacuum, the photons are not, in the same way, a primary physical reality, but are generated as opportune excitations - associated to the non-null frequency given by (9) - of this special state of the $3 \mathrm{D}$ quantum vacuum. It is functioning as a reservoir of photons, under opportune conditions, namely when they correspond to the minimum "quantum" of the electromagnetic properties of the vacuum characterized by a quantized vector potential. The latter depends on the quantum vacuum energy density fluctuations and the number of the RS processes in the volume under consideration.

In summary, we can say that, in our model at the fundamental level, our physical world emerges from the $3 \mathrm{D}$ non-local quantum vacuum characterized by elementary RS processes of creation/annihilation of virtual particle/antiparticle pairs corresponding to elementary fluctuations of the quantum vacuum energy density. Those fluctuations are choreographed by the quantum potential (8) of the vacuum, as well as by the field $\boldsymbol{\Xi}_{0}$ which depends on a complex unit vector of polarization (which expresses the polarization features of a region of the 3D quantum vacuum) and represents the ground state of the $3 \mathrm{D}$ quantum vacuum which acts as a reservoir of photons. The primary non-local features of the $3 \mathrm{D}$ vacuum, as well as its polarization features, imply that physical photons emerge from this ground state of the $3 \mathrm{D}$ quantum vacuum, intended as a sort of the reservoir in such a way that, in this fundamental background, there is a universal interconnection of things - and thus also of potential photons - which could no longer be questioned. Thus, the photons themselves are not independent of this fundamental background, but form an indivisible system with the background. This implies that the processes of entrance and exit of physical photons from the vacuum are nucleation processes which occur instantaneously at a distance one with another, in a compatible way with the original Wheeler-Feynman absorber theory and the transactional approach. In other words, we can say that the non-locality of the 3D quantum vacuum characterized by polarization features has the important role to explain the physical appearance of photons from the ground state acting as a reservoir of photons in terms of medium's excitations which must be seen as timesymmetric processes. In them, one has an "absorber" which generates confirmation waves in response to an emitted offer wave, in agreement with the WheelerFeynman absorber theory and the transactional approach. The non-locality of the $3 \mathrm{D}$ quantum vacuum makes the nucleation processes of photons as physical processes which occur instantaneously at a distance.

Moreover, as a consequence of the field $\boldsymbol{\Xi}_{0}$ acting as a reservoir of photons, the following treatment of the spontaneous emission of photons by matter emerges in the suggested $3 \mathrm{D}$ quantum vacuum model. In the epistemological affinity with Meis [2829 ], the electron-vacuum interaction can be described by considering a "vacuum action" operator corresponding to a Hamiltonian interaction per angular frequency between the vacuum field $\boldsymbol{\Xi}_{0}$ and an atomic electron of mass $m_{e}$ and charge $e$, where the electron itself is determined by opportune RS processes 
of creation/annihilation of virtual particles of the 3D quantum vacuum. This Hamiltonian interaction per angular frequency can be expressed as follows:

$H_{\frac{\Delta \rho_{q \nu E}}{n}}=-i \hbar \frac{e}{m_{e}} \boldsymbol{\Xi}_{0} \cdot \nabla$.

Here, taking Melis' results into account, if one calculates the amplitude of the transition probability per angular frequency of an atom between the given initial state and a certain final state in the presence of $n$ photons, one finds

$W_{i f}=\frac{1}{3 \hbar c^{2}} \frac{e^{2}}{4 \pi \varepsilon_{0}} \omega_{i f}^{3}\left|\mathbf{r}_{i f}\right|^{2} d \Omega$.

Result (27) turns out to be in agreement with the previous QED calculations [30-32], as well as with Meis' treatment [28-29], and shows that the spontaneous emission of photons takes place by starting from the ground state of the frequency of the $3 \mathrm{D}$ quantum vacuum through the action of the creation operator $a_{k, \lambda}^{+}$, which is involved in the quantum vacuum expression. In other words, the vacuum field $\boldsymbol{\Xi}_{0}$ can be considered as a sort of the "bare" skeleton structure of every physical photon of the world, which ultimately emerges from the RS processes of creation/annihilation of virtual particles from the 3D quantum vacuum which perturbs this "bare" skeleton vacuum state by conferring it frequency (9), when there is an interaction with matter.

\section{Derivation of Planck's}

\section{Law of Traditional Black-Body Spectrum}

In the original Planck theory, the spectral distribution of the energy radiated by a black body follows a law based on the introduction of quanta of energy $\hbar \omega$, whose nature is not better specified, except for the fact that it represents a necessary mathematical artifice to provide a correct statistical treatment. In Planck's original theory, in fact, the hypothesis of the quantization of oscillators' energy allowed Planck to integrate the results obtained respectively by Wien for small wavelengths and by Rayleigh-Jeans for large wavelengths in a single coherent picture, starting from considerations on the nature of the entropy of the radiation. As a consequence, the Planck spectrum of the black-body radiation is usually presented by the major part of textbooks without accounting for the zero-point radiation part (see [33-36]). In the standard view, in the light of the experimental work by
Lummer and Pringsheim [37], the Planck energy for a harmonic oscillator is given by the formula

$U_{P}(\omega, T)=\frac{\hbar \omega}{\exp \left[\frac{\hbar \omega}{K_{\mathrm{B}} T}\right]-1}$.

The radiation emitted by a black body that ranges from $\omega$ to $\omega+d \omega$ contributes on the average, to the energy field within a volume $\mathrm{dV}$, an energy that is proportional to $d \omega$ and $d V$ expressed by (e.g., Döring [38], Landau \& Lifshitz [39])

$d E=\frac{\hbar}{\pi^{2} c^{3}} \frac{\omega^{3}}{\exp \left[\frac{\hbar \omega}{K_{\mathrm{B}} T}\right]-1} d \omega d V$

where the quantity

$U(\omega, T)=\frac{\omega^{3}}{\exp \left[\frac{\hbar \omega}{K_{\mathrm{B}} T}\right]-1}$

is the monochromatic (or spectral) energy density of radiation which, according to Planck's approach, in the case of thermal equilibrium, is related to the average energy of a harmonic oscillator of the frequency $\omega$ located inside the cavity walls (Planck [40], Pauli [41]).

Instead, in our three-dimensional quantum vacuum approach, the quanta of energy hypothized by Planck as regards the spectral distribution of the radiation of a black body are derived from more fundamental processes associated with the energetic fluctuations of the three-dimensional quantum vacuum. The quanta of energy $\hbar \omega$ emerge from energy fluctuations of the $3 \mathrm{D}$ quantum vacuum on the basis of the fundamental relations (9) regarding the radiation associated with the elementary RS processes and the quantization volume (24) responsible for the appearance of photons.

As we have seen in the previous section, the photon is an emerging entity from more fundamental properties of the 3D quantum vacuum, inside a minimum spatial extension corresponding to the quantization volume (24) in which the quantized vector potential (17) of the $3 \mathrm{D}$ quantum vacuum oscillates over a period. This means that Planck's quanta of energy of the black-body spectrum, as well as Planck's law of the spectral distribution of the energy radiated by a black body, ultimately follow from this fundamental 
property of the quantization volume of the 3D quantum vacuum.

In order to provide this derivation, we consider some fruitful considerations made recently by T.H. Boyer in the context of his derivations of the Planck black-body spectrum within classical physics, where he applied the thermodynamic smoothness required by the first and second laws of thermodynamics, in a picture, where the thermal radiation, zero-point radiation, and spacetime structure are all tightly related [42]. By applying the $1^{\text {st }}$ and $2^{\text {nd }}$ laws of thermodynamics to a harmonic oscillator or a radiation normal mode, the canonical potential of a harmonic oscillator in Boyer's approach is a function of the single variable corresponding to the ratio of the oscillation frequency to the temperature. The notion of thermodynamic smoothness requires that the canonical potential of a harmonic oscillator neither distinguish any frequency $\omega$ at a given temperature $T$, nor any temperature at a given frequency. At a minimum, the notion of smoothness demands that any interpolation function for the canonical potential of the oscillator and all its derivatives be monotonic; the monotonic behavior prevents a single value for $\omega / T$ from being distinguished by the canonical potential. By choosing the needed small interpolation function as

$\phi_{\text {Pzp }}(\omega, T)=-\ln \{2 \sinh [\omega /(2 T)]\}$,

the associated energy can be directly expressed as

$U_{\mathrm{Pzp}}(\omega, T)=T^{2}\left(\frac{\partial \phi_{\mathrm{Pzp}}}{\partial t}\right)=\frac{\omega}{2}+\frac{\omega}{\exp (\omega / T)-1}$,

which corresponds exactly to the Planck formula including the zero-point energy.

Now, in our model of the 3D quantum vacuum defined by a fundamental variable energy density, it is possible to provide a new re-reading to the results of Boyer, in the sense that Eqs. (31) and (32) may be seen as structures emerging from more fundamental processes governing the regions of the $3 \mathrm{D}$ quantum vacuum. In other words, Planck's law of the spectral distribution of the energy radiated by a black body ultimately derives from regions of the 3D quantum vacuum, when it is characterized by the ground state defined by Eqs. (25) and (26), namely when it acts as a "reservoir" of photons. The latter themselves appear in the context of a minimum spatial extension corresponding to the quantization volume (24) in which the quantized vector potential (17) of the 3D quantum vacuum oscillates over a period. In our approach, the frequency of a oscillator is determined by more fundamental fluctuations of the quantum vacuum energy density. Therefore, in the light of Boyer's considerations deriving from the $1^{\text {st }}$ and $2^{\text {nd }}$ laws of thermodynamics with regard for the interpolation function for the canonical potential of the oscillators of the black body spectrum, we can say that the regions of 3D quantum vacuum acting as a reservoir of photons must have the property to do not distinguish any fluctuation of the quantum vacuum energy density at a given temperature nor any temperature at a given quantum vacuum energy density. This requirement means that the regions of the $3 \mathrm{D}$ quantum vacuum originating photons can be described by a function of the form

$\phi_{\operatorname{Pzp}}\left(\frac{\Delta \rho_{q \nu E}}{n}, T\right)=-\ln \left\{2 \sinh \left[\frac{2 \Delta \rho_{q \nu E}}{\hbar n} /(2 T)\right]\right\}$.

Here, since photons appear in the quantization volume (24), the variable quantum vacuum energy density satisfies the condition

$\left(\frac{2 \Delta \rho_{q \nu E}}{\hbar n}\right)=\left(\frac{\hbar}{2 \varepsilon_{0} \xi^{2} V}\right)^{1 / 3}$.

Therefore, the function of the vacuum potential (33) can be also written as

$\phi_{\mathrm{Pzp}}(V, T)=-\ln \left\{2 \sinh \left[\left(\frac{\hbar}{2 \varepsilon_{0} \xi^{2} V}\right)^{1 / 3} /(2 T)\right]\right\}$.

On the basis of Eqs. (33) and (35), one can obtain directly the associated energy of the $3 \mathrm{D}$ quantum vacuum which originates the spectral distribution of Planck's law of spectral distribution of the energy radiated by a black body:

$U_{\text {Pzp }}\left(\frac{\Delta \rho_{q \nu E}}{n}, T\right)=T^{2}\left(\frac{\partial \phi_{\mathrm{Pzp}}}{\partial t}\right)=$

$=\frac{\Delta \rho_{q \nu E}}{\hbar n}+\frac{\frac{2 \Delta \rho_{q \nu E}}{\hbar n}}{\exp \left(\frac{2 \Delta \rho_{q \nu E}}{\hbar n T}-1\right)}$,

namely,

$U_{\text {Pzp }}(V, T)=\frac{1}{2}\left(\frac{\hbar}{2 \varepsilon_{0} \xi^{2} V}\right)^{1 / 3}+$

$+\frac{\left(\frac{\hbar}{2 \varepsilon_{0} \xi^{2} V}\right)^{1 / 3}}{\exp \left(\left(\frac{\hbar}{2 \varepsilon_{0} \xi^{2} V}\right)^{1 / 3} / T\right)-1}$. 
In our theory, Eqs. (36) and (37) express the fundamental property of the $3 \mathrm{D}$ quantum vacuum to generate the spectral distribution of the black body radiation in agreement with Planck's original treatment. Here, the oscillators of Boyer's approach, which turn out to have the behavior described by Eqs. (31) and (32) derive from more fundamental regions of the $3 \mathrm{D}$ quantum vacuum described by Eqs. (33), (35), (36), and (37).

Moreover, always on the basis of Boyer's considerations, the entropy of the regions of the $3 \mathrm{D}$ quantum vacuum acting as reservoirs of photons depends on the variable quantum vacuum energy density on the basis of the relation

$S\left(\frac{\Delta \rho_{q \nu E}}{n}, T\right)=-\ln \left\{2 \sinh \left[\frac{\Delta \rho_{q \nu E}}{\hbar n T}\right]\right\}+$

$+\frac{\Delta \rho_{q \nu E}}{\hbar n T} \operatorname{coth}\left[\frac{\Delta \rho_{q \nu E}}{\hbar n T}\right]$

namely,

$$
\begin{aligned}
& S(\omega, T)=-\ln \left\{2 \sinh \left[\left(\frac{\hbar}{2 \varepsilon_{0} \xi^{2} V}\right)^{1 / 3} /(2 T)\right]\right\}+ \\
& +\frac{2}{T}\left(\frac{\hbar}{2 \varepsilon_{0} \xi^{2} V}\right)^{1 / 3} \operatorname{coth}\left[\left(\frac{\hbar}{2 \varepsilon_{0} \xi^{2} V}\right)^{1 / 3} /(2 T)\right] .
\end{aligned}
$$

It must be emphasized that the entropy is a monotonic function of $U / \frac{\Delta \rho_{q \nu E}}{n}$, and all the derivatives are monotonic functions, in agreement with Boyer's results.

\section{3D Quantum Vacuum}

\section{as a Reservoir of Photons and its Role with regard for the Lamb shift of Hydrogen-Like Atoms}

By starting from the zero-point energy density defining the electromagnetic effects of the $3 \mathrm{D}$ quantum vacuum expressed by relation (10), we can consider a spectral radiation density

$W_{\rho_{q \nu E}}=\frac{1}{8 \pi} E_{\rho_{q \nu E}}^{2}$.

Thus,

$E_{\rho_{q \nu E}}^{2}=64 \frac{\left(\Delta_{\rho_{q \nu E}}\right)^{3} V^{3} Q_{Q, i}}{\hbar^{3} n^{3} c^{3}}$

which, by applying condition (24), becomes

$E_{\rho_{q \nu E}}^{2}=4 \frac{\hbar V^{2} Q_{Q, i}}{\varepsilon_{0} \xi^{2} c^{3}}$

116
On the basis of Eq. (42), it is possible to obtain a derivation of the Lamb shift of hydrogenoid atoms in a picture, where the $3 \mathrm{D}$ quantum vacuum is a cosmic reservoir of photons in the ground state represented by Eqs. (25) and (26) in the context of a minimum spatial extension corresponding to the quantization volume (24) in which the quantized vector potential (17) of the 3D quantum vacuum oscillates over a period.

In this regard, first of all, we observe that, in the first approximation, the energy level displacements of the electron bounded states can be seen as a topological effect of the vacuum radiation pressure exerted upon the electronic orbitals. In fact, the RS processes of the vacuum determining the appearance and the corresponding motion of a bound-state electron in a hydrogenoid atom generates a quantized vector potential of the form (17) in the surrounding region of the $3 \mathrm{D}$ quantum vacuum. This leads, in turn, to the vacuum field $\boldsymbol{\Xi}_{0}$ given by Eqs. (25) and (26) involving the polarization and generating the electronic transitions in matter. Hence, we have the photons of energy $\hbar \omega$ in this region of the vacuum, which are just derived from energy fluctuations of the 3D quantum vacuum on the basis of the fundamental relations (9) regarding the radiation associated with the elementary RS processes and in the context of the minimum quantized space defined by condition (24).

Now, because of the periodic motion of the RS processes (associated with the appearance of an electron) inside the field $\boldsymbol{\Xi}_{0}$, these RS processes feel the influence of the photons generated by the vacuum. This interaction between the RS processes of the electron and the photons of the vacuum can be described by considering the pressure per unit surface

$d P($ vacuum $)=\Sigma_{\lambda} \varepsilon_{0} E_{\rho_{q \nu E}}^{2} d \Omega$

With regard for the results obtained in our recent paper [43], as a consequence of the interaction with the photons generated by the ground state of the 3D quantum vacuum, the motion of an electron turns out to be determined by fluctuations obeying the equation

$\ddot{s}_{\rho_{q \nu E}}=\left(\frac{16 c^{3} \varepsilon_{0} \xi^{2} V}{\hbar^{4} n^{3}}\right)^{1 / 3} \sqrt{\frac{\hbar V^{2} Q_{Q, i}}{\varepsilon_{0} \xi^{2} c^{3}}} \cos \left[\left(\frac{\hbar}{2 \varepsilon_{0} \xi^{2} V}\right)^{1 / 3} t\right]$.

ISSN 2071-0194. Ukr. J. Phys. 2020. Vol. 65, No. 2 
The effect of a fluctuation $s_{\rho_{q \nu E}}$ is to generate a change $\Delta V$ in the average potential

$\Delta \Phi=\Phi\left(r+s_{\rho_{q \nu E}}\right)-\Phi(r)$,

where $V\left(r+s_{\rho_{q \nu E}}\right)$ is computed by Taylor's expansion:

$\Phi\left(r+s_{\rho_{q \nu E}}\right)=\Phi(r)+\frac{\partial \Phi}{\partial s_{\rho_{q \nu E}}} s_{\rho_{q \nu E}}+$

$+\frac{1}{2} \sum_{i j} \frac{\partial^{2} \Phi}{\partial s_{\rho_{q v E, i}} \partial s_{\rho_{q v E, j}}} s_{\rho_{q v E, i}} s_{\rho_{q v E, j}}+\ldots$.

Now, by averaging expression (46) in time, one observes that the second term vanishes because $\mathbf{s}$ averages to zero. For the same reason, only the contributions with $i=j$ remain in the final term. As a consequence, after some mathematical manipulations, the following expression for a change in the energy for the hydrogenoid atoms holds:

$\delta W_{\rho_{q \nu E}}=\pi e^{2} s_{\rho_{q \nu E}}^{2}\left\langle n^{\prime}, l^{\prime}, m^{\prime}|\delta(r)| n, l, m\right\rangle$.

Now, since the matrix element (47) provides contributions only for $\mathrm{S}$ states, where its value is

$\left|\psi_{100}(r, \vartheta, \varphi)\right|^{2}=\frac{Z^{3}}{\pi n^{3} a_{0}^{3}}$,

by inserting (45) and (48) into (47), we get

$\delta W_{\rho_{q \nu E}}=\frac{e^{10} Z^{4} V^{2 / 3}}{2^{4 / 3} 3 \pi \varepsilon_{0}^{4 / 3} n \hbar^{11 / 3} c^{5} \xi^{2 / 3}}$.

Now, integrating over the two cut-off values of the quantized volume corresponding, respectively, to the quantum vacuum energy density associated with the orbiting electron and with the rest energy of the electron, we obtain

$\delta W=\frac{1}{5} \frac{e^{10} Z^{4} V^{2 / 3}}{2^{4 / 3} \pi \varepsilon_{0}^{4 / 3} n \hbar^{11 / 3} c^{5} \xi^{2 / 3}}\left[\left(V_{\max }^{5 / 3}\right)-\left(V_{\min }^{5 / 3}\right)\right]$,

which is the expression of the Lamb shift predicted by the ground state of the $3 \mathrm{D}$ quantum vacuum acting as a reservoir of photons.

Let us remember now that, in the original QED approach developed by Welton in [44] and Weisskopf in [45], the corresponding result for the Lamb shift was

$\delta W=\frac{4}{3 \pi} \alpha^{3} \frac{Z^{4}}{n^{3}} \ln \left(\frac{\nu_{\max }}{\nu_{\min }}\right)$,

ISSN 2071-0194. Ukr. J. Phys. 2020. Vol. 65, No. 2 where $\nu_{\min }$ is the frequency of an orbiting electron $\left(\frac{Z^{2}}{n^{3}}\right.$ in atomic units), and $\nu_{\max }$ corresponds to the rest energy of the electron, namely, $\frac{\nu_{\max }}{\nu_{\min }} \approx \frac{Z^{2}}{n^{3} \alpha^{2}}$. For the $2 \mathrm{~S}$ state, we have

$\delta W=\frac{1}{6 \pi} \alpha^{3} \ln \left(\frac{8}{\alpha^{2}}\right)=1.600 \mathrm{MHz}$.

As a consequence, by comparing (50) and (51), one has the possibility to obtain the opportune quantum vacuum energy density fluctuations of the RS processes which generate the frequencies of the orbiting electron and of the rest energy of the electron, respectively:

$$
\begin{aligned}
& \frac{4}{3 \pi} \alpha^{3} \frac{Z^{4}}{n^{3}} \ln \left(\frac{\nu_{\max }}{\nu_{\min }}\right)= \\
& =\frac{1}{5} \frac{e^{10} Z^{4}}{2^{4 / 3} \pi \varepsilon_{0}^{4 / 3} n \hbar^{11 / 3} c^{5} \xi^{2 / 3}}\left[\left(V_{\max }^{5 / 3}\right)-\left(V_{\min }^{5 / 3}\right)\right],
\end{aligned}
$$

namely,

$\ln \left(\frac{\nu_{\max }}{\nu_{\min }}\right)=\frac{3}{20} \frac{e^{10} Z^{4} n^{2}}{2^{4 / 3} \varepsilon_{0}^{4 / 3} \hbar^{2 / 3} c^{2} \xi^{2 / 3}}\left[\left(V_{\max }^{5 / 3}\right)-\left(V_{\min }^{5 / 3}\right)\right]$

and

$\ln \nu_{\max }-\ln \nu_{\min }=$

$=\frac{3}{20} \frac{e^{10} Z^{4} n^{2}}{2^{4 / 3} \varepsilon_{0}^{4 / 3} \hbar^{2 / 3} c^{2} \xi^{2 / 3}}\left[\left(V_{\max }^{5 / 3}\right)-\left(V_{\min }^{5 / 3}\right)\right]$.

Thus,

$\ln \nu_{\max }=\frac{3}{20} \frac{e^{10} Z^{4} n^{2}}{2^{4 / 3} \varepsilon_{0}^{4 / 3} \hbar^{2 / 3} c^{2} \xi^{2 / 3}}\left(V_{\max }^{5 / 3}\right)$

and

$\ln \nu_{\min }=\frac{3}{20} \frac{e^{10} Z^{4} n^{2}}{2^{4 / 3} \varepsilon_{0}^{4 / 3} \hbar^{2 / 3} c^{2} \xi^{2 / 3}}\left(V_{\min }^{5 / 3}\right)$.

Equations (56) and (57) constitute the bridge between our model of the 3D quantum vacuum energy density and the original Welton's and Weisskopf's model and assure the compatibility of our model with the results obtained in the context of QED. If conditions (56) and (57) are satisfied, the model of the 3D quantum vacuum developed in this section allows us to obtain the Lamb shift of the hydrogenoid atoms 
in agreement with relation (51) and, thus, with the original Welton's and Weisskopf's model.

On the basis of Eqs. (53)-(57), the following rereading of the Lamb shift origin becomes permissible. The frequencies associated with the orbiting electron and with the rest energy of the electron of hydrogenoid atoms (given by the original Welton's and Weisskopf's model) are produced by the regions of the $3 \mathrm{D}$ quantum vacuum acting as a source originating photons inside a minimum spatial extension corresponding to the quantization volume (24) in which the quantized vector potential (17) of the $3 \mathrm{D}$ quantum vacuum oscillates over a period. Equations (53)(57) express, in what sense the 3D quantum vacuum acting as a reservoir of photons is the primary physical reality which is responsible for the Lamb shift of hydrogenoid atoms, by relating the frequencies associated with the orbiting electron and with the rest energy of the electron of hydrongoid atoms with the quantization volume in which the quantized vector potential of the 3D quantum vacuum oscillates over a period, as well as other parameters characteristic of the region of the $3 \mathrm{D}$ quantum vacuum generating the photons. It must be emphasized that, in our approach, the results are in agreement with the results obtained in the conventional renormalization method of QED.

\section{Conclusions}

Although quantum field theory has lead to the idea of that the physical vacuum is endowed with an intrinsic energy density corresponding to a sort of the polarization, the Standard Model does not seem to have learned this lesson and states that the particles and fields exist in an "empty space". As a consequence, it is not possible in this picture to have a clear understanding from where a photon appears and where it disappears. In the model suggested in this paper, through elementary processes regarding the timeless 3D quantum vacuum, the electron is just a "door" through which photon enters into existence and a door through which a photon turns back into the quantum vacuum. In particular, the ground state describing the electromagnetic properties of the $3 \mathrm{D}$ quantum vacuum represents the ultimate source from which photons are born: if subatomic massive particles are ultimately generated by more elementary physical processes regarding the RS processes of the $3 \mathrm{D}$ quantum vacuum, the photons are not a primary physical reality in the same way, but are generated by a special state of the $3 \mathrm{D}$ quantum vacuum, which is functioning as a reservoir of photons under opportune conditions.

The model where the quantum vacuum is the primordial arena of the Universe turns out to be compatible with the foundations of the original WheelerFeynman absorber theory, as well as of the most recent developments regarding the transactional interpretation. At the same time, by introducing the interesting novel perspectives concerning the re-reading of the origin of photon, it has the immense potential for the development of various spheres of theoretical physics.

1. A. Sorli, U. Dobnikar, S.K. Patro, M. Mageshwaran, D. Fiscaletti. Euclidean-Planck Metrics of Space, Particle Physics and Cosmology. NeuroQuantology 16, 18 (2018).

2. Stanford Solar Center, http://solar-center.stanford.edu/ comics/ref/em-sunspectrum.pdf (2017).

3. J. Wheeler, R. Feynman. Interaction with the absorber as the mechanism of radiation. Rev. Mod. Phys. 17, 157 (1945).

4. J.G. Cramer. Generalized absorber theory and the Einstein-Podolsky-Rosen paradox. Physical Review D 22, 362 (1980).

5. J.G. Cramer. The arrow of electromagnetic time and the generalized absorber theory. Foundations of Physics 13, 887 (1983).

6. J.G. Cramer. The transactional interpretation of quantum mechanics. Rev. Mod. Phys. 58, 647 (1986).

7. J.G. Cramer. An overview of the transactional interpretation. Int. J. Theor. Phys. 27, 27 (1988).

8. R.E. Kastner. De Broglie waves as the "bridge of becoming" between quantum theory and relativity. Foundations of Science 18, 1 (2013).

9. R.E. Kastner. On delayed choice and contingent absorber experiments. ISRN Mathematical Physics 2012, Article ID 617291 (2012).

10. R.E. Kastner. The broken symmetry of time. AIP Conference Proceedings 1408, 7 (2011).

11. R.E. Kastner. The New Transactional Interpretation of Quantum Theory: The Reality of Possibility (Cambridge Univ. Press, 2012).

12. L. Chiatti. The transaction as a quantum concept. In: Space-Time Geometry and Quantum Events, edited by I. Licata (Nova Sci., 2014), pp. 11-44; e-print arXiv.org/pdf/1204.6636 (2012).

13. I. Licata. Transaction and non-locality in quantum field theory. European Physical Journal Web of Conferences (2013).

14. I. Licata, L. Chiatti. The archaic universe: big bang, cosmological term and the quantum origin of time in projective cosmology. Int. J. Theor. Phys. 48, 1003 (2009).

ISSN 2071-0194. Ukr. J. Phys. 2020. Vol. 65, No. 2 
15. I. Licata, L. Chiatti. Archaic universe and cosmological model: 'big-bang' as nucleation by vacuum. Int. J. Theor. Phys. 49, 2379 (2010).

16. I. Licata, L. Chiatti. Timeless approach to quantum jumps. Quanta 4, 10 (2015).

17. L. Chiatti, I. Licata. Particle model from quantum foundations. Quantum Studies: Mathematics and Foundations 4, 191 (2017).

18. D. Fiscaletti, A. Sorli. Perspectives about quantum mechanics in a model of a three-dimensional quantum vacuum where time is a mathematical dimension. SOP Transactions on Theoretical Physics 1, 11 (2014).

19. D. Fiscaletti, A. Sorli. Space-time curvature of general relativity and energy density of a three-dimensional quantum vacuum. Annales UMCS Section AAA: Physica LXIX, 55 (2014).

20. D. Fiscaletti. The Timeless Approach. Frontier Perspectives in $21^{\text {st }}$ Century Physics (World Scientific, 2015).

21. D. Fiscaletti, A. Sorli. About a three-dimensional quantum vacuum as the ultimate origin of gravity, electromagnetic field, dark energy ... and quantum behavior. Ukr. J. Phys. 61, 13 (2016).

22. D. Fiscaletti, A. Sorli. Dynamic quantum vacuum and relativity. Annales UMCS Section AAA: Physica LXXI, 11 (2016).

23. D. Fiscaletti, What is the actual behavior of the electron? From Bohm's approach to the transactional interpretation. . . to a three-dimensional timeless non-local quantum vacuum. Electronic J. Theor. Phys. 13, 1 (2016).

24. D. Fiscaletti, A. Sorli. Quantum vacuum energy density and unifying perspectives between gravity and quantum behavior of matter. Annales de la Fondation Louis de Broglie 42, 251 (2017).

25. D. Fiscaletti, A. Sorli. Quantum relativity: variable energy density of quantum vacuum as the origin of mass, gravity and the quantum behavior. Ukr. J. Phys. 63, 623 (2018).

26. D. Fiscaletti, A. Sorli. Bijective epistemology and spacetime. Foundations of Science 20, 387 (2015).

27. D. Fiscaletti, A. Sorli. Searching for an adequate relation between time and entanglement. Quantum Studies: Mathematics and Foundations 4, 357 (2017).

28. C. Meis. Vector potential quantization and the quantum vacuum. Physics Research International 2014, Article ID 187432 (2014).

29. C. Meis. The nature of the photon and the quantum vacuum http://www.mse-chair.org/wp-content/uploads /2017/03/Photons-Vacuum by-C.MEIS .pdf (2018).

30. B.H. Bransden, C.J. Joachain. Physics of Atoms and Molecules (Longman, 1983).

31. H. Haken. Light (North Holland, 1981).

32. M. Weissbluth. Photon-Atom Interactions (Academic Press, 1988).
33. See, for example: R. Eisberg, R. Resnick. Quantum Physics of Atoms, Molecules, Solids Nuclei, and Particles (Wiley, 1985).

34. K.S. Krane. Modern Physics (Wiley, 1996).

35. J.R. Taylor, C.D. Zafiratos, M.A. Dubson. Modern Physics for Scientists and Engineers (Pearson, 2003).

36. S.T. Thornton, A. Rex. Modern Physics for Scientists and Engineers (Brooks/Cole, 2013).

37. O. Lummer, E. Pringsheim. Die Vertheilung der Energie im Spectrum des schwarzen Köpers und des blanken Platins. Verhandlungen der Deutschen Phyikalischen Gesellschaft 1, 215 (1899)

38. W. Döring. Atomphysik und Quantenmechanik: I. Grundlagen (Walter de Gruyter, 1973).

39. L.D. Landau, E.M. Lifshitz. Course of Theoretical Physics. Vol. 5: Statistical Physics (Pergamon Press, 1980)

40. M. Planck. Ueber das Gesetz der Energieverteilung im Normalspectrum. Ann. d. Physik 4, 553 (1901).

41. W. Pauli. Statistical Mechanics (MIT Press, 1973).

42. T.H. Boyer. Derivation of the Planck blackbody spectrum from thermodynamic ideas in classical physics with classical zero-point radiation. arXiv:1802.04653v1 [physics.genph] (2018).

43. D. Fiscaletti, A. Sorli. About the hydrogenoid atoms in the timeless three-dimensional quantum vacuum. Quantum Studies: Mathematics and Foundations, accepted for publication, 2019.

44. T.A. Welton. Some Observable Effects of the Quantum Mechanical Fluctuations of the Electromagnetic Field. Phys. Rev. 74, 1157 (1948).

45. V.F. Weisskopf. Recent developments in the theory of the electron. Rev. Mod. Phys. 21, 305 (1949).

Received 13.06.19

Д. Фіскалетті, А. Сорлі

ТРИВИМІРНИЙ НЕЛОКАЛЬНИЙ

КВАНТОВИЙ ВАКУУМ ЯК ДЖЕРЕЛО ФОТОНІВ

$\mathrm{P}$ е $з$ ю м е

Запропоновано модель тривимірного квантового вакууму, що визначається процесами народження/анігіляції квантів, які відповідають елементарним флуктуаціям щільності енергіі. У цій моделі фотон не є вихідним фізичним об'єктом, але виникає як особливий стан тривимірного квантового вакууму. В основному стані вакуум є " космічним резервуаром", який випромінює і поглинає фотони. Спектральний розподіл Планка для енергії, що випромінює чорне тіло, є наслідком фундаментальних процесів у тривимірному квантовому вакуумі, зокрема, в аспекті квантування об'єму, відповідального за появу фотонів. Досліджується уявлення про лембовський зсув у водневоподібних атомах як явищі, що визначається основним станом квантового вакууму як резервуара фотонів. 